Horizons philosophiques

\title{
Petites nations et républicanisme
}

\section{Du multiculturalisme à l'auto-gouvernement}

\section{Dave Anctil}

Volume 13, numéro 2, printemps 2003

Herder (1744-1803) : le clair-obscur

URI : https://id.erudit.org/iderudit/801239ar

DOI : https://doi.org/10.7202/801239ar

Aller au sommaire du numéro

Éditeur(s)

Collège Édouard-Montpetit

\section{ISSN}

1181-9227 (imprimé)

1920-2954 (numérique)

Découvrir la revue

Citer cet article

Anctil, D. (2003). Petites nations et républicanisme : du multiculturalisme à

l'auto-gouvernement. Horizons philosophiques, 13(2), 93-113.

https://doi.org/10.7202/801239ar d'utilisation que vous pouvez consulter en ligne.

https://apropos.erudit.org/fr/usagers/politique-dutilisation/ 


\section{PETITES NATIONS ET RÉPUBLICANISME. DU MULTICULTURALISME À L'AUTO-GOUVERNEMENT'}

Plusieurs arguments militent en faveur de la reconnaissance de droits collectifs à des groupes minoritaires et/ou vulnérables de la société. Les limites du cadre libéral classique des droits et libertés individuels apparaissent aussi de plus en plus aux philosophes, juristes et politologues contemporains, principalement à la lumière d'une compréhension plus franche et plus nuancée des constructions sociales et culturelles de nos sociétés pluralistes. Mais des problèmes majeurs apparaissent lorsque certains groupes minoritaires sur un territoire se considèrent comme des "nations". Cette situation (un nombre $X$ de nations pour un nombre $X$ - d'États souverains) devient de plus en plus difficile à justifier, car plusieurs de ces nations minoritaires revendiquent aussi le droit d'exercer une autonomie politique sur un territoire (et ainsi de cesser d'y être minoritaires). Une multitude d'arguments normatifs et pratiques leur donnent raison. Pourtant, malgré la multiplication des États souverains au cours du $X X e$ siècle, il existe toujours une forte opposition à ces mouvements autonomistes dont une bonne part sont aussi résolument indépendantistes.

Dans ce texte nous présentons une défense normative et pratique de la quête d'autonomie politique des petites nations ${ }^{2}$. Cependant, nous choisissons de construire une argumentation qui se distingue en plusieurs points du cadre démocratique libéral traditionnel. En effet, l'autonomie politique d'une nation implique selon nous une multitude d'institutions et de pouvoirs qu'une simple politique libérale de la reconnaissance n'est pas en mesure de fournir, surtout parce qu'elle laisse ouverte la possibilité de dominations internes et externes (par exemple de la part de l'État central dans le cas d'une fédération). Voilà aussi pourquoi nous choisissons le cadre de la théorie politique normative républicaine, principalement représentée aujourd'hui par le philosophe Philip Pettit. Nous appliquons dans ce texte cette conception républicaine au problème des minorités nationales en quête d'auto-gouvernement, afin de voir en quoi cette perspective particulière peut nous permettre de justifier la volonté autonomiste des petites nations. 
Nous développons cette position en trois étapes : nous débutons par une présentation générale et contextuelle du débat entourant la construction nationale des États. Ensuite, dans une comparaison critique avec la perspective républicaine, nous identifions quelques limites du cadre démocratique libéral actuel pour surmonter le problème que posent les petites nations autonomistes. Enfin, nous tentons de former une justification spécifiquement républicaine de l'auto-gouvernement, conçue comme une alternative plus sensible à la question de la vulnérabilité des groupes nationaux minoritaires en quête d'autonomie politique.

\section{La construction nationale des États et le retour des «petites nations"}

L'écroulement de l'URSS et les luttes de décolonisation se sont traduits par une montée fulgurante et revendicatrice des nationalismes minoritaires. L'intelligentsia occidentale a reçu ces mouvements de manière paradoxale. Elle a d'une part condamné les crimes subis par les petits peuples sous le joug des grandes puissances et a d'autre part été terriblement critique face aux revendications autonomistes de ces minorités. Les intellectuels, souvent proches idéologiquement des grands centres du pouvoir mondial, sont restés habituellement peu sensibles aux particularités des revendications autonomistes des petits peuples ${ }^{3}$. Les élites des puissances occidentales voyaient en ces nouveaux nationalismes une sorte de "tribalisme" ou encore un essentialisme romantique 4 . Alain Finkielkraut reprenait à ce sujet une polémique brillamment engagée par l'écrivain tchèque Milan Kundera, qui disait, au sujet de la petite nation, qu'elle était "celle dont l'existence peut être à n'importe quel moment remise en question, qui peut disparaître et le sait5". II ne s'agit pas ici d'une définition prégnante au niveau normatif, mais elle donne à penser ce que Kymlicka appelle "l'ethnocentrisme" des grandes nations à l'égard des minorités nationales ${ }^{6}$. Cette définition est aussi importante pour comprendre la conscience collective petite-nationale, au caractère "périssable" et "précaire». Composée de dizaines de millions d'individus, partageant une langue et une histoire communes universellement reconnues, une armée puissante et des institutions centenaires, la "grande nation" (ou encore la "nation parvenue") ne manifeste apparemment que très peu de complexes identitaires. Elle ne se sent jamais sérieusement menacée de disparition. Ses craintes se situent ailleurs. Maintenir par exemple 
le statu quo ante qui assure sa puissance, un sentiment normal dirions-nous, un simple "patriotisme" de bon aloi...

Pour ce qui est du fameux terme de "nationalisme", on choisit le plus souvent de laisser le fâcheux épithète aux extrémistes de la droite ethnique réactivant un discours xénophobe teinté d'impérialisme du XIXe siècle, ou encore, ironiquement!, au supposé "tribalisme" de certains membres des petites nations ${ }^{7}$. Cette distinction (entre un sentiment d'appartenance "légitime» et un autre qui serait «illégitime») oublie une longue histoire de conquêtes et de colonialisme, le plus souvent justifiée par la grandeur inhérente au «progrès» qu'incarnerait l'État moderne.

Or la situation contemporaine devrait nous conduire à questionner ces "certitudes" pour plusieurs raisons. On peut raisonnablement considérer aujourd'hui que l'époque des empires et des conquêtes semble bel et bien terminée. La pacification relative du monde politique contemporain résulte en partie d'un "consensus" presque mondial au sujet des droits humains - consensus dépendant, il est vrai, de la puissance d'un Occident plus unifié politiquement et économiquement. Les discours politiques ainsi que les modalités d'action des entités politiques ne peuvent tout simplement plus se justifier à partir d'une simple «raison d'État» transcendante; cette dernière fait place à la nécessité pour l'État de donner aujourd'hui des "raisons" pour justifier ses actions dans l'espace public international8. De sorte que le dépassement de l'ancien "état de nature» interétatique diminue considérablement la liberté des États - une licence autrefois mesurée simplement en terme de puissance.

Au début du siècle passé, il y avait à peu près 40 pays souverains dans le monde, alors qu'on en dénombre aujourd'hui à peu près $185^{9}$. Cette nouvelle réalité géopolitique contemporaine est d'une importance capitale pour comprendre la transformation des relations entre les États et leurs minorités nationales ${ }^{10}$. Les petites nations étaient autrefois condamnées aux décisions arbitraires de ces centres parfois très éloignés; ces mêmes centres autoritaires doivent maintenant faire face à la volonté nouvellement légitimée des revendications autonomistes des minorités nationales sur «leurs» territoires. Celles-ci profitent admirablement bien de la décomposition rapide des super-États du passé et sont aujourd'hui en mesure de présenter des requêtes ou de rechercher toujours plus d'autonomie sans subir de répression excessive.

Mais pour répondre de la validité normative de ces droits 
collectifs, et dont l'ultime manifestation est l'auto-gouvernement, il nous faut d'abord donner une définition satisfaisante de la "nation" lorsque celle-ci ne possède pas son propre État (ou en possède un incomplet). Un certain consensus semble émerger au sujet de la distinction entre la nation, comme communauté politique particulière, et l'État, en tant qu'il est l'autorité juridique, politique et administrative souveraine et légitime sur un territoire particulier. Pour les théories fondatrices de l'Etat moderne, Hobbes, Kant, Locke, Hegel et Weber restent des incontournables. Seulement, cette tradition vénérable reste le plus souvent impuissante à penser de manière "claire et distincte" les rapports entre les minorités nationales et l'organisation étatique de la société : cette tradition assume sans la questionner la construction nationale historique des États. Voilà pourquoi nous choisissons de nous limiter à la littérature contemporaine qui prend le parti d'une analyse à prétention normative, c'est-à-dire qui construit les «idéals-types" que sont l'État et la nation en vue d'en arriver à des consensus rationnels au sujet de la justice - sans prétendre à la perfection descriptive et/ou qualitative des "réalités" de ces concepts.

Historiquement, l'État s'organise en effet en fonction des intérêts de la majorité nationale sur son territoire. La domination de cette majorité fait en sorte que l'État prend de plus en plus l'image de celle-ci, et cela très souvent au mépris de l'existence d'autres nations sur son territoire. Ce processus, appelé "Nation-building", vise à construire l'unité du pays, c'est-à-dire à lui donner un ordre, des standards communs (linguistiques, culturels, législatifs et administratifs) qui visent à assurer une solidarité citoyenne minimale ${ }^{11}$. Les groupes minoritaires sont le plus souvent ignorés, voire volontairement discriminés, pendant la construction nationale du pays, même s'ils constituent des "cultures sociétales" distinctes de la majorité. Dans les mots de Kymlicka, les individus à l'intérieur de ces groupes forment une :

culture qui offre à ses membres des modes de vie, porteurs de sens, qui modulent l'ensemble des activités humaines, au niveau de la société, de l'éducation, de la religion, des loisirs et de la vie économique, dans les sphères publique et privée. Ces cultures tendent à être territorialement concentrées et fondées sur une communauté linguistique.

Je dis de ces cultures qu'elles sont «sociétales» pour souligner 
le fait qu'elles ne renvoient pas simplement à une mémoire ou à des valeurs partagées, mais comprennent en outre des institutions et des pratiques communes ${ }^{12}$.

Cette définition est très importante parce qu'elle permet de distinguer au moins trois formes de groupe, et ensuite de montrer la spécificité de la nation en tant qu'elle est une culture sociétale : (1) Les nations, y compris les petites, sont donc des «sociétés distinctes et potentiellement aptes à exercer une certaine autonomie gouvernementale au sein d'un État». (2) Les "groupes ethniques" forment pour leur part des collectivités plus ou moins cohérentes d' «immigrants qui ont quitté leur communauté nationale pour s'installer dans une autre société». (3) Enfin, les "groupes de mouvements sociaux» recoupent «les associations et les mouvements formés par les homosexuels, les femmes, les pauvres, les personnes handicapées" historiquement «marginalisés» 13 .

Ces distinctions permettent à Kymlicka de renverser l'argument de la "diversité des groupes» qui vise à miner la légitimité des réclamations des minorités nationales au nom de l'égalité individuelle. Les États sont en effet très souvent de facto des États multinationaux (mais de jure des États mono-nationaux), et très souvent des États poly-ethniques. Tous les États modernes composent aussi avec des groupes de mouvements sociaux qui ont des réclamations dans l'espace public. Ces mêmes États utilisent alors les groupes les uns contre les autres en affirmant ne pas vouloir privilégier personne. Cette stratégie, qui s'appuie sur la thèse libérale de la supposée «neutralité bienveillante» de l'État, désavantage particulièrement les petites nations parce qu'elle ne reconnaît pas leurs besoins spécifiques - et souvent plus substantiels ${ }^{14}$. Pourtant, si les droits avantagent effectivement des individus à l'intérieur des États libéraux, c'est bien parce que ces mêmes individus jouissent d'un statut particulier commun, nommément celui de citoyen. La conséquence est donc que tous les droits politiques ou civiles sont donc d'emblée des droits de groupes, car ils sont déjà présupposés, même en régime libéral, dans la forme politique d'un monde divisé en différents «groupes-États»15.

Au niveau pratique, la mondialisation des marchés économiques vient de plus diminuer considérablement les avantages liés au fait d'appartenir à de grands États. Dans ce contexte, on ne voit plus très bien ce qui, a priori du moins, devrait empêcher les minorités 
nationales de remettre en question les règles et les normes intégrées aux liens politiques obligés avec ces grands États. C'est justement la thèse du livre de Stéphane Paquin qui vient donner une légitimité pratique à notre justification de l'autonomie des petites nations : «la mondialisation, en diminuant les avantages de l'intégration et les obstacles à l'indépendance, favorise la désintégration (des grands États) ${ }^{16}$ ".

Ce développement n'implique pas cependant que le Nationbuilding soit injustifiable en lui-même. La preuve étant que les petites nations essaient aussi par tous les moyens de se donner des politiques de construction nationale afin d'entrer en compétition, aux niveaux symbolique et politique, avec les États tutélaires. II y a plusieurs raisons à cela. Premièrement, le système international ou "système westphalien", c'est-à-dire inter-étatique ou "stato-national" (il n'admet d'autorité territoriale qu'aux États souverains), ne laisse pas beaucoup de choix aux nations en quête d'une plus grande autonomie politique : ces dernières essaient plus ou moins d'obtenir l'indépendance politique, c'est-à-dire la reconnaissance de la souveraineté d'un État (le leur) sur le territoire «national». Cette situation pourrait changer dans le futur sous les effets transformateurs de la mondialisation. Mais pour l'instant, le système de l'État-nation domine intégralement - d'où l'objectif maintenu de la souveraineté étatique ${ }^{17}$.

Deuxièmement, et plusieurs auteurs commencent de plus en plus à le reconnaître, le modèle de l'État-nation n'est pas étranger à la réalisation ou du moins à la consolidation de la démocratie libérale ${ }^{18}$. Comme le remarque Kymlicka en réponse au cosmopolitisme utopique de David Held : "there are good reasons to think that territorialized linguistic / national political units provide the best and perhaps the only sort of forum for genuinely participatory and deliberative politics ${ }^{19}$ ".

Troisièmement, la consolidation des petites entités nationales, c'est-à-dire la réalisation croissante de leur autonomie pouvant mener jusqu'à l'indépendance politique, est peut-être valable en elle-même pour des questions de justice. Si nous acceptons l'idée que les droits collectifs accordés à des minorités nationales visent à réparer ou à minimiser des situations désavantageuses pour les membres de ces groupes, il se peut alors que l'auto-gouvernement, la forme suprême de reconnaissance d'un groupe, soit un impératif moral afin de donner les moyens à de petites nations de prospérer librement20. 


\section{Quelques limites du cadre libéral classique : une critique républicaine}

Nous espérons jusqu'à maintenant avoir bien souligné que : 1) les groupes nommés "nations" sont distincts des États souverains eux-mêmes; 2) que les minorités nationales à l'intérieur des pays doivent être distinguées des autres groupes de la société ayant des prétentions aux droits collectifs (groupes ethniques d'immigration et mouvements sociaux); 3) que plusieurs raisons de justice militent en faveur de l'octroi de droits collectifs aux groupes défavorisés par la construction nationale des États qui, historiquement, ont favorisé les majorités nationales sur leur territoire; 4) ensuite, que ces droits existaient déjà implicitement dans le système politique international, puisque seuls des "citoyens", et non pas simplement des «individus" au sens abstrait, peuvent bénéficier de facto de droits significatifs dans les démocraties libérales existantes; 5 ) enfin, que l'autonomie nationale peut avoir une valeur politique (elle donne une liberté étatique sur un territoire), une valeur démocratique (elle permet à une citoyenneté effective de prendre forme) et une valeur morale (elle promeut la justice, l'égalité et la liberté).

Nous aimerions maintenant émettre quelques réserves d'ordres politiques et institutionnels à l'égard du cadre libéral21. Ces critiques nous inciteront par la suite à formuler une justification républicaine du droit à l'autonomie politique22.

Premièrement, le libéralisme classique s'appuie le plus souvent sur une justification individualiste (et jusnaturaliste) des droits et libertés, ce qui explique ses réticences quasi-naturelles par rapport à la reconnaissance de droits collectifs. Nous avons montré qu'il était cependant possible de défendre des droits de groupe dans le cadre d'une théorie libérale, par exemple celle de Kymlicka. Néanmoins, cette théorie libérale du multiculturalisme s'appuie sur un individualisme moral problématique et les droits de groupe qu'elle reconnaît entrent en tension perpétuelle avec les droits individuels 23 . La position républicaine que nous défendons reconnaît pour sa part le principe de "pertinence individuelle» (individual relevance), qui permet d'évaluer les impacts des institutions sur les individus ${ }^{24}$. $\mathrm{Ce}$ principe n'implique pas a priori une forme d'individualisme moral - il n'implique pas qu'un groupe doive exister, légitimement et de manière instrumentale, uniquement pour les individus - mais bien que les justifications collectives de droits, de libertés ou d'institutions soient 
"pertinentes" (relevant) à l'égard des individus de ce groupe 25 . Ce principe ne pourrait pas permettre, par exemple, un droit de groupe qui n'aurait aucun avantage/impact (à court ou long terme) sur les individus composants ce groupe. II s'accorde avec l'idée générale suivante: "Pour qu'un groupe social puisse réclamer des droits collectifs, il doit jouer un rôle important dans l'individuation de toute personne (membre) ${ }^{26}$ ". Car il ne faut pas oublier que les biens promus par des droits collectifs ne sont pas les propriétés d'individus, mais ils ont néanmoins un impact sur les individus. Cette thèse s'oppose donc à tout collectivisme qui ne prendrait pas en considération l'impact sur les individus27.

Deuxièmement, la théorie libérale s'intéresse en priorité à la justification morale des droits collectifs, au même titre qu'elle le fait pour les droits individuels. La seconde critique républicaine porte sur le fait que la position libérale classique devient alors souvent insensible aux types d'instances qui confèrent ces droits dans le régime politique. Elle peut très bien par exemple s'accommoder d'un pouvoir discrétionnaire qui pourrait intervenir de manière arbitraire; pourvu que les "droits individuels" soient respectés, cette situation serait juste. Dans ce cadre libéral, même l'empire ou une certaine forme de douce tyrannie étrangère se justifient. La liberté libérale n'implique pas en ce sens l'auto-gouvernement. C'est justement là qu'est la difficulté : il arrive très souvent que l'instance qui garantit le droit à l'intérieur de l'État soit en mesure d'abroger et/ou de modifier les droits accordés aux membres de la minorité nationale28. Par exemple, la théorie libérale peut très bien accepter que, dans la pratique, des droits linguistiques soient reconnus (ou simplement tolérés) à la nation québécoise par l'État fédéral central, tout en maintenant les prérogatives qui lui permettraient de suspendre et/ou d'invalider ces droits en temps de crise ou lorsque le gouvernement de cet État jugerait que ceux-ci contreviennent aux droits individuels 29 . Dans le cadre d'une théorie républicaine, de tels droits sont problématiques parce qu'ils sont soumis à la bonne volonté d'un pouvoir qui peut choisir d'intervenir arbitrairement. Selon nous, de tels droits doivent se fonder sur un socle d'inviolabilité ou encore, dans le cas de biens particuliers, sur une assurance institutionnelle à l'abri de la puissance arbitraire de l'État et du gouvernement (ce qui inclut parfois les tribunaux eux-mêmes). Pour des républicains, en effet, cette assurance prend la forme de l'auto-gouvernement, une condition nécessaire (mais non suffisante) à la liberté. 
Troisièmement, la théorie libérale classique ne fait pas de la promotion citoyenne des droits et libertés une nécessité politique. En effet, les libéraux s'en remettent souvent trop facilement à la loi seule pour protéger les droits reconnus. Or il est évident que la loi, même abrogée ou améliorée, demeure souvent impuissante à remplir les exigences impliquées par les droits reconnus juridiquement ${ }^{30}$. Dans le cadre d'une théorie républicaine, ces droits doivent se lier avec l'idée même de citoyenneté active: les citoyens forment alors une vigile constante et s'assurent de l'application et de l'effectivité de leurs droits, qu'ils soient individuels ou collectifs - il en va ainsi de leur responsabilité civique. Le républicanisme conçoit aussi les institutions de l'État comme encourageant cette participation citoyenne au renforcement des lois, des droits et des libertés à l'intérieur de la communauté politique ${ }^{31}$. Ses institutions doivent être marquées par la transparence, l'accessibilité et la participation différenciée dans une optique de maximisation du principe de publicité. Les droits sont renforcés par les citoyens; inversement, la citoyenneté est renforcée par les droits.

Enfin, la perspective libérale uniquement centrée sur les droits et libertés (individuels ou collectifs) est insuffisante politiquement. Dans la perspective libérale, rien n'empêche a priori que les droits soient octroyés par un pouvoir étranger, c'est-à-dire indirectement ${ }^{32}$. À l'opposé, la théorie républicaine ne peut se contenter de la simple reconnaissance de droits aux individus et aux groupes. Elle exige de plus que ces derniers aient les pouvoirs nécessaires pour faire respecter ces droits et réaliser les biens que ces droits sont censés entraînés. Sans ces pouvoirs politiques, les groupes et les individus ne sont pas en mesure de défendre leurs droits contre des interventions potentiellement arbitraires. Ces menaces sont en général des formes de domination contre lesquelles les lois et les institutions républicaines permettent aux citoyens, ainsi qu'à leurs représentants, de se protéger ${ }^{33}$. Le républicanisme que nous défendons conçoit les droits et libertés, ainsi que les pouvoirs légaux qu'ils impliquent, comme étant imbriqués dans la citoyenneté. Pour le dire autrement et de manière républicaine, la communauté politique historique, et non une dignité humaine abstraite et universelle, est la source véritable de ce «statut spécial» qu'est celui du citoyen jouissant de droits juridiques et de la liberté politique - d'où l'idée centrale de souveraineté du peuple ${ }^{34}$. 
Cette critique en quatre temps forme ainsi un argument en faveur de l'autonomie politique des petites nations non souveraines. Elle implique une promotion de l'autonomie nationale à quatre niveaux : 1) Niveau moral : la justification de droits ne s'appuie pas sur un individualisme moral ou méthodologique; elle reconnaît aussi l'importance des collectivités sans réduction collectiviste. 2) Niveau institutionnel : des droits effectifs impliquent une protection institutionnelle autonome des groupes contre tout pouvoir arbitraire. 3) Niveau de la citoyenneté : la promotion et la réalisation des droits impliquent une citoyenneté active ("contestataire"), elle instaure les mécanismes d'une protection mutuelle des droits et libertés; les droits et libertés sont conçus comme imbriqués dans la citoyenneté elle-même. 4) Niveau politique : les droits et les institutions impliquent donc que soient alloués des pouvoirs suffisants à la communauté afin de se protéger contre des pouvoirs extérieurs qui la menacent.

Nous avons vu que la petite nation qui recherche l'autonomie politique ne peut pas faire confiance aux pouvoirs extérieurs à elle-même afin de promouvoir et de défendre les droits, les libertés et les intérêts communs de ses membres. Nous définissons aussi ce nationalisme (ou patriotisme) en général comme le sentiment d'un engagement responsable envers la communauté nationale, qui implique la promotion et la protection de la communauté (langue, culture, institutions, etc.), et laquelle suppose un vouloir-vivre collectif national35. Cela implique-t-il que les nations, même et surtout les petites, doivent obtenir nécessairement des États indépendants? Le plus souvent, l'accommodation est le choix le plus avisé politiquement, afin d'éviter le conflit ou encore par crainte d'instabilités socio-économiques. Cependant, lorsque l'autonomie politique complète (interne et externe) est possible, la souveraineté étatique devient selon nous le choix le plus avisé36.

Une légitimation normative républicaine porte cette réalité à un niveau plus fondamental: selon nous, l'autonomie politique d'une nation implique une série d'institutions et de pouvoirs qu'une simple politique de la reconnaissance n'est pas en mesure de fournir. Dans la définition républicaine des droits et libertés, la domination réelle ou potentielle que représente l'État central, sa situation de fiduciaire juridique envers la petite nation, est inacceptable. Comme nous le voyons, le républicanisme implique aussi que nous définissions différemment les droits et libertés, les institutions politiques ainsi que le sens de l'autonomie politique - ce qu'il nous faut montrer dès maintenant. 


\section{Liberté républicaine et auto-gouvernement}

Nous choisissons d'adopter le concept de liberté républicaine selon la définition donnée par le philosophe Philip Pettit dans son maître-livre : Republicanism - A Theory of Freedom and Government. La liberté politique républicaine est d'abord une situation effective de non-domination, c'est-à-dire que des pouvoirs et des mécanismes institutionnels protégent les individus contre l'interférence arbitraire dans leurs choix ${ }^{37}$. La domination peut correspondre en effet à une multitude d'interférences (physiques, psychologiques, institutionnelles, administratives, etc.). Mais ces interférences, pour être jugées "dominantes", doivent ensuite partager un caractère arbitraire, c'est-à-dire qu'elles dépendent de l'arbitratum d'un agent (individuel ou collectif), de sa volonté, d'une décision qu'il est en mesure de réaliser. Une telle intervention peut aussi être "sélective" ou limitée, sans nécessairement impliquer une interférence dans tous les choix possibles mais uniquement dans certains. Enfin, les pouvoirs qui rendent possible la domination sont de toutes sortes, mais ils représentent toujours des avantages particuliers dont jouissent certains agents individuels ou collectifs 38 .

Une autre caractéristique centrale impliquée dans la domination est son caractère intersubjectif (ou psychologique), comme par exemple ce que Kundera et Finkielkraut disaient au sujet de la vulnérabilité consciente de la petite nation. Pettit l'explique ainsi : «Domination is generally going to involve the awareness of control on the part of the powerful, the awareness of vulnerability on the part of the powerless, and the mutual awareness - indeed, the common awareness among all the parties to the relationship - of this consciousness on each side ${ }^{39}$ ". C'est pourquoi le républicanisme se définit aussi et en général comme une "culture de la liberté", intolérante envers toute culture de la soumission et de la servitude: elle ne peut tolérer que des citoyens de la nation vivent dans la peur, l'exclusion et la subordination, parce qu'une telle existence serait un affront général aux principes constitutifs de la liberté générale ${ }^{40}$.

Cette culture de la liberté et cette détestation de la domination font en sorte que les manifestations de cette dernière se doivent d'être reconnues comme étant des maux graves contre lesquels les institutions républicaines servent de rempart. La liberté comme nondomination est donc un bien commun premier de la citoyenneté ${ }^{41}$. Au niveau individuel, elle implique la disparition des conditions censées 
diminuer nos possibilités positives d'action; la liberté personnelle repose ainsi sur l'absence d'ingérence arbitraire, aussi bien éventuelle qu'effective, de la part d'autrui. Nous voyons plus loin qu'il en est de même au niveau collectif.

Cependant, il est important de comprendre que l'interférence en elle-même n'est pas nécessairement une forme de domination (i.e. n'est pas nécessairement arbitraire). Nous expliquions plus haut, en effet, que les lois justes et consenties ont précisément pour but de nous rendre libres en mettant en place des barrières contre la volonté arbitraire des autres. Plus limitée, la conception libérale d'une liberté comme "non-interférence» propose une théorie du droit qui se représente toute loi comme une restriction artificielle de la liberté. Pour des républicains, argumente Pettit, l'interférence ne sera pas arbitraire (donc non-dominante et compatible avec la liberté) si et seulement si sa relation vise la poursuite des intérêts pertinents et volontaires de l'individu ou du groupe qui en est l'objet ${ }^{42}$. Voilà aussi pourquoi une vie politique active et un espace public dynamique sont nécessaires à la recherche commune et concertée de la liberté.

Les institutions républicaines mettent de l'avant deux stratégies principales afin de contrer la domination 43 : 1) La stratégie des pouvoirs réciproques, qui vise à donner des pouvoirs à tous les citoyens, c'est-à-dire les moyens de contrer ou de contrebalancer des pouvoirs naturellement inégalitaires par une égalisation de statut (distincte de la "neutralité étatique» et de l'«égalité des chances» libérales); 2) La stratégie des provisions constitutionnelles, qui introduit une autorité constitutionnelle dans la société afin de contrer les possibilités de domination de la part des mieux lotis. Ces stratégies institutionnelles ne visent pas à rendre les agents individuellement ou collectivement impuissants, mais bien à leur donner quelque chose comme un contre-pouvoir. Source de liberté, ces institutions représentent un contrôle qu'une personne ou un groupe bénéficie lorsqu'il est protégé afin d'orienter sa destinée comme il l'entend et en toute sécurité 44 . Ce pouvoir est précieux et doit être compris comme un statut à la fois politique, social et légal : celui que confère, au niveau collectif, l'auto-gouvernement, et au niveau individuel, la citoyenneté45. Un tel statut confère le bien social et politique le plus précieux, c'est-à-dire une liberté comme non-domination pour tous.

Cette présentation sommaire complétée, nous nous intéressons dans cet article à l'application de la théorie républicaine au problème des minorités nationales en quête d'autonomie, afin de voir en quoi 
cette perspective différente, complémentaire mais aussi critique, peut nous permettre de justifier la volonté autonomiste des petites nations. Or Pettit ne traite presque pas de ces problèmes dans son Republicanism. II aborde cependant brièvement la question plus générale du multiculturalisme, en affirmant que cet idéal de tolérance s'accorde en général avec l'idéal républicain de liberté comme non-domination ${ }^{46}$. Le républicanisme doit en effet reconnaître les demandes des minorités culturelles et la liberté comme nondomination implique de donner les moyens aux groupes vulnérables de ne pas être dominés. Comme le remarque le philosophe :

(...) so far as membership in a minority culture is likely to be a badge of vulnerability to domination, the members of that culture, and the state that assumes concern for their fortunes, must address the needs of the culture in general. It is not going to be enough to claim to be concerned with individuals in the culture, without any particular reference to what binds them together ${ }^{47}$.

Cet argument défend l'idée générale de la valeur du groupe, à savoir que les groupes sont aussi sujets à être dominés, et que c'est en tant que groupes (par exemple une culture sociétale minoritaire ou nation minoritaire), qu'ils doivent pouvoir jouir de droits particuliers et de ressources leur assurant une situation de non-domination 48 . Cette reconnaissance implique aussi un argument en faveur de l'autonomie politique. En effet, selon Pettit, la responsabilité de la majorité nationale à l'égard d'une minorité nationale peut aller jusqu'à la reconnaissance de l'indépendance politique ou du moins, si cela n'est pas possible ou non voulu, d'une autonomie suffisante pour le groupe en question 49 . Cet appui général à l'idée de "citoyenneté multiculturelle" correspond donc à l'idéal républicain de liberté comme nondomination, car la théorie républicaine reconnaît aussi l'importance politique des cultures sociétales.

Or il est impératif de noter que l'importance du groupe que Pettit reconnaît est beaucoup plus significative au niveau politique ${ }^{50}$. Une telle appartenance n'est pas seulement et simplement une affiliation culturelle générale (importante du point de vue de la non-domination), mais bien une affiliation particulière des membres d'un groupe particulier ${ }^{11}$. Puisque le fait de jouir d'une situation permanente de non-domination exige d'être dans une position dans laquelle personne ne puisse intervenir de manière arbitraire, cela implique, mutatis 
mutandis, que personne ne puisse intervenir arbitrairement dans les affaires d'un groupe partageant la même vulnérabilité. En d'autres mots, la stratégie républicaine rend compte, individuellement, de l'affiliation particulière à un groupe parce qu'elle reconnaît que la vulnérabilité est une caractéristique commune comme le sont les autres attributs ou caractéristiques positives qui le définissent. Voilà aussi pourquoi elle recommande la solidarité à l'intérieur du groupe ${ }^{52}$.

II ne faut pas oublier le fait qu'une véritable situation de nondomination exige une protection contre les interventions arbitraires potentielles et effectives. C'est pourquoi la vulnérabilité des intérêts de certains groupes devient aussi une caractéristique du groupe lui-même. Par exemple, une petite nation sujette à l'autorité d'un gouvernement extérieur à elle peut se trouver dans une situation globalement avantageuse et cette autorité peut la traiter globalement avec respect et dignité. Seulement, la minorité nationale n'est pas alors dans une situation de liberté au sens républicain: son bonheur dépend de la bonne volonté d'un État, c'est-à-dire d'un agent extérieur à elle qui pourra toujours, par exemple lors d'un changement de gouvernement, lui faire regretter sa situation de dépendance ${ }^{53}$. Tant que la domination reste possible dans le régime politique, c'està-dire tant que la minorité nationale peut en tant que groupe être la victime d'une contrainte arbitraire, cette nation et les membres qui la composent se trouvent de facto et de jure dominés - donc non-libres au sens républicain du terme. Politiquement, cela signifie qu'une nation minoritaire qui n'a pas la souveraineté reconnue sur son territoire restera toujours sous le joug d'un pouvoir potentiellement arbitraire. On ne peut parler face à face sans un statut égal (donc légal) reconnu, et les recours d'un État sont de fait plus nombreux et beaucoup plus imposants que toute autre forme de statut politique et juridique, car ce statut donne une personnalité juridique dans l'organisation politique internationale.

La situation de non-domination nécessite donc une modification des conditions de la relation. Elle requiert l'inaccessibilité d'une interférence arbitraire et pas seulement son improbabilité. Cette modification des conditions de la relation doit aussi nécessairement se faire à un niveau collectif (at the level of group grievance and group assertion), et pas seulement à celui des individus ${ }^{54}$. La recherche et la réalisation des conditions de non-domination est donc un argument puissant en faveur de l'autonomie politique des petites nations vulnérables. Cet argument synthétique peut prendre la forme 
suivante 55 : Considérant que les nations forment des groupes distincts ayant des besoins particuliers qui impliquent aussi des droits particuliers; Que ces besoins et ces droits sont liés aux caractéristiques particulières qui définissent ces groupes nationaux; Que ces besoins et ces caractéristiques collectives sont vulnérables à la construction nationale des États tutélaires ainsi qu'à la démocratie majoritaire; Que cette situation (potentielle ou réelle) de vulnérabilité correspond à une forme collective de domination (accessible et probable); Que les effets de cette domination provoquent des préjudices et une situation d'injustice (comme l'inégalité de statut collectif qui entraîne des conséquences négatives multiples); Que cette situation collective de domination empêche aussi les individus de jouir individuellement et collectivement d'une liberté comme nondomination (parce que leur situation de vulnérabilité devient aussi une caractéristique de tous les individus du groupe national en question); Que l'autonomie politique est la forme privilégiée de droit collectif, au sens où elle permet par la suite une quantité non limitée (i.e. auto-limitée) de mesures institutionnelles favorisant la protection et la promotion du groupe national; Que l'État, enfin, demeure la seule forme politique reconnue de souveraineté territoriale au niveau international, et que les groupes nationaux se trouvent le plus souvent sur un territoire particulier; Alors l'autonomie politique, préférablement la souveraineté étatique de la nation sur son territoire, est à la fois la situation la plus juste, mais aussi la plus désirable socialement et politiquement.

Peut-être que le phénomène de la mondialisation modifiera significativement un jour les privilèges accordés aux États et favorisera ainsi, dans le futur, des formes d'auto-gouvernement qui n'impliqueront pas la lourdeur administrative du modèle stato-national. Pour l'heure, en tout cas, la souveraineté étatique sur un territoire demeure d'une importance capitale pour assurer une liberté citoyenne significative aux individus appartenant à de petites nations.

\section{Conclusion}

Nous avons tenté de présenter une justification normative et pratique en faveur de l'auto-gouvernement des minorités nationales, et essayé de présenter nos arguments dans un cadre néo-républicain qui se voulait critique du cadre libéral traditionnel au sujet des droits collectifs et de la liberté politique. Ce choix fut motivé avant tout par des considérations stratégiques. En effet, nous voulions proposer une 
argumentation qui différait des justifications libérales qui tendent souvent à s'éloigner du langage politique lui-même, lui préférant un langage moral trop limité et peu efficace dans l'espace public.

Dans une perspective républicaine, la valeur de l'autonomie politique, pour reprendre le trait de la pensée de Hannah Arendt, se traduit en effet par la capacité d'agir en commun et ainsi d'engendrer du pouvoir, capacité qui est fondée sur le vouloir-vivre collectif et régie par un lien institutionnellement reconnu. Ce lien politique, celui que confère une communauté politique historique envers laquelle il est possible de s'identifier et à l'intérieur de laquelle il est possible de participer, est selon nous plus fondamental et plus durable que le fait de la domination et de l'isolement. En effet, le lien de solidarité que crée le combat pour la liberté commune est du domaine de l'expérience et défiera probablement toujours l'explication rationnelle. Mais comment dénigrer la valeur du lien réciproque qui existe entre notre communauté nationale d'appartenance et la citoyenneté qu'elle rend possible et effective?

La liberté est effective uniquement lorsqu'elle est partagée; la conquête de la liberté est aussi une entreprise politique commune qui solidifie les liens d'appartenance. Comme le remarquait James Tully au sujet de la lutte des peuples autochtones : ce combat pour la liberté devient ainsi un combat de liberté. S'il peut aujourd'hui prendre place dans le cadre de la démocratie, s'il peut faire l'économie d'une lutte armée et utiliser les "armes" de la parole et de l'argument, alors le rôle de l'intellectuel et du philosophe en particulier est évident et ce dernier ne pourra pas toujours faire abstraction de sa situation particulière. S'il croit que la liberté est une conquête politique significative et désirable, il doit mettre en œuvre les moyens de la réaliser. Telle est du moins la perspective de la tradition républicaine, qui dira toujours avec Tocqueville : "Qui cherche la liberté pour autre chose qu'elle-même, est fait pour servir."

\section{Dave Anctil lord_nurgle@hotmail.com}

1. Nous tenons à remercier le professeur Michel Seymour de l'Université de Montréal pour l'inspiration initiale de cet essai qui a pris naissance dans son séminaire sur les droits collectifs. Merci aussi à la professeure Linda Cardinal, de I'Université d'Ottawa, pour ses excellentes critiques. Enfin, à notre collègue et ami Mathieu Bock-Côté, pour ses recommandations particulières. 
2. Pour des raisons de simplicité, notre argumentation portera spécifiquement sur les cas des nations autonomistes non autochtones (Québec, Catalogne, Écosse, etc.). Nous croyons néanmoins que l'essentiel de notre propos s'appliquerait aux cas des nations autochtones en quête d'autonomie politique, mais nous n'avons pas l'espace ici pour développer cette position - qui impliquerait, pour reconnaître la spécificité autochtone, des précisions substantielles. Par exemple, les nations autochtones ne veulent pas, le plus souvent, d'un État indépendant. Voir James Tully, "The Strugles of Indigenous Peoples for and of Freedom", in Political Theory and the Rights of Indigenous Peoples, D. Ivison, P. Patton \& W. Sanders (dirs.), Cambridge, 2000, p. 36-59; et James S. Anaya, Indigenous Peoples in International Law, Oxford, 2000.

3. Ce que Michael Keating a justement appelé le "chauvinisme métropolitain» des intellectuels qui a «largement contribué à légitimer les entreprises de l'État-nation». Voir Les défis du nationalisme moderne. Québec, Catalogne, Écosse, Presses de I'Université de Montréal, 1997, p. 36.

4. Alain Finkielkraut, "Qu'est-ce qu'une petite nation?», in M. Seymour (dir.), Nationalité, citoyenneté et solidarité, 1999, p.435-461.

5. Milan Kundera, "Un Occident kidnappé, la tragédie de l'Europe centrale", Le débat, no 27, novembre, 1983.

6. Voir La citoyenneté multiculturelle, Boréal, 1995, p. 111-112; 139-148.

7. Pour cette distinction abusivement aveuglée par l'évolution historico-politique des nations européennes, voir l'essai par ailleurs intéressant de Maurizio Viroli : For Love of Country - An Essay on Patriotism and Nationalism, Oxford ( $\left.2^{\mathrm{e}} \mathrm{ed}.\right)$, 1997.

8. Dominique Schnapper, La communauté des citoyens - Sur l'idée moderne de nation, Gallimard, 1994, p. 198-202.

9. Stéphane Paquin, La revanche des petites nations - Le Québec, l'Écosse et la Catalogne face à la mondialisation, VLB Éditeur, 2001, p.16.

10. Voir David Laitin, "National Identities in the Emerging European State», in Minority Nationalism and the Changing International Order, Oxford, p. 84-113.

11. Voir Kymlicka, Politics in the Vernacular - Nationalism, Multiculturalism and Citizenship, Oxford, 2001, p. 26; David Miller, Citizenship and National Identity, Polity Press, 2000, p. 88-89.

12. Kymlicka, La citoyenneté multiculturelle, p. 115.

13. Ibid., p. 36.

14. Voir Kymlicka, Ibid., p. 78, 155-158, 184; et Politics in the Vernacular, p. 23-24.

15. La citoyenneté multiculturelle, p. 181.

16. Stéphane Paquin, La revanche des petites nations, p. 20. Voir aussi Christian Rioux, Voyage à l'intérieur des petites nations, Boréal, 2000, p. 183; Kymlicka, Politics in the Vernacular, p. 246, 345.

17. Dans le cas des minorités nationales européennes, l'intégration économique et politique de l'Europe permet d'espérer que celles-ci auront une occasion de gagner en autonomie politique sans nécessairement avoir à rechercher la souveraineté étatique sur leur territoire au sens classique du terme (voir Neil MacCormick, "Liberalism, Nationalism and the Post-Sovereign State", in R. Bellamy \& D. Castiglione (dirs.), Blackwell, 1996 p.141-155). Une des possibilités pour 
ces nations réside dans le "principe de subsidiarité" qui gagne rapidement en popularité et qui pourrait s'avérer positif à une plus grande autonomie politique des minorités nationales (pour un résumé de ce concept, voir Andreas Föllesdal, "Survey Article : Subsidiarity", The Journal of Political Philosophy, vol. 6, n० 2, 1998, p.190-208.

18. Voir Yaël Tamir, Liberal Nationalism, Princeton UP, 1993; Alan Patten, "The Autonomy Argument for Liberal Nationalism", Nations and Nationalim, 5,1, 1999 et "Conception libérale de la citoyenneté et identité nationale, in Seymour (dir.), Nationalité, citoyenneté et solidarité», Ibid.; Margaret Canovan, Nationalism and Political Theory, Elgar, 1996.

19. Kymlicka, Politics in the Vernacular, p. 319-322. Voir aussi Miller, Citizenship and National Identity.

20. Cependant, cette affirmation n'implique pas l'exigence excessive de légitimité que l'on rencontre souvent dans la littérature théorique à ce sujet. Allen Buchanan, par exemple, semble vouloir associer trop facilement les revendications de reconnaissance juridique des nations à un "droit à la souveraineté» unilatérale (voir "Les conditions de la sécession", in M. Seymour (dir.), Une nation peut-elle se donner la constitution de son choix?, Bellarmin, 1995, p. 170). Or ce n'est pas nécessairement le cas. À en croire la logique de Buchanan, il ne suffirait même pas à une nation d'être moralement irréprochable pour être justifiée d'accéder à l'indépendance politique; il lui faudrait souffrir suffisamment! En effet, sa théorie du «droit à la sécession" ne reconnaît que les cas de "réparations historiques", sans reconnaître le droit à l'autonomie alloué présentement dans le cadre du droit international... (Voir "The International Institutional Dimension of Secession", in P. B. Lehning, Theories of Secession, Routledge, 1998, p. 236-239.)

21. Ces critiques s'inspirent entre autres des textes de $P$. Pettit qui soulignent les limites politiques de la théorie libérale : "The Freedom of the City : A Republican Ideal», in The Good Polity, Blackwell, 1989, p. 141-168; "The Normative Analysis of the State : Some Preliminaries", in The Good Polity, Ibid. p. 1-13; The Common Mind - An Essay on Psychology, Society and Politics, Oxford, 1993; "Freedom as Antipower", Ethics, avril 1996, p. 576-604; "Reworking Sandel's Republicanism", Journal of Philosophy, 95, 1998, pp.73-96; Republicanism - A Theory of Freedom and Government, Oxford ( $2^{\mathrm{e}}$ ed.) 1999; et de Quentin Skinner, Liberty Before Liberalism, Cambridge, 1998.

22. Notons que la théorie néo-républicaine de Pettit recherche, au niveau théorique, le même objectif d' "équilibre réflexif» que Rawls ( $A$ Theory of Justice, Harvard ( ${ }^{e}$ ed.), 1971 (voir p. 18-19, 42-45, 507-508). Pettit prescrit deux conditions minimales pour que la philosophie politique s'approche de cet équilibre : une assomption personnaliste (les objets des institutions politiques doivent être des êtres humains), et une assomption universaliste (les accords doivent être satisfaisants au niveau normatif pour tous les individus) (voir Pettit, The Common Mind, p. 286-287; Republicanism, p. 96.

23. Kymlicka marque lui-même la différence et veut être clair là-dessus : «From a liberal egalitarian point of view, communal identities and civic virtues can only play a secondary role, to be judged by the extent to which they are consistent with, or promote, foundational values of individual agency and social justice." (Politics in the Vernacular, p. 332) Dans cette philosophie libérale pourtant amendée, la liberté politique, impliquant l'auto-gouvernement et la vertu civique, demeure donc toujours instrumentalisée. Voir par exemple les précisions de Kymlicka, La citoyenneté 
multiculturelle, p. 72-76. Nous limitons notre critique de l'individualisme libéral à la perspective (politico-institutionnelle) républicaine. Mais pour une critique plus générale : «les arguments individualistes en faveur de l'importance de l'affiliation culturelle ne parviennent pas à démontrer que cette affiliation linguistique, culturelle ou nationale, en tant qu'affiliation particuliere, est importante, mais bien que le fait d'avoir une telle affiliation, peu importe laquelle, est très important." (Seymour, "Qui a peur des droits collectifs?", Terminogramme, 95-96, 2001, p. 45-49; voir aussi La nation en question, l'Hexagone, 1999, p. 144 et "Quelques aspects politiques de l'anti-individualisme", in Une nation peut-elle se donner la constitution de son choix ?, Ibid.).

24. La stratégie républicaine rend compte en effet de l'affiliation particulière, parce qu'elle reconnaît que ce sont des caractéristiques communes au groupe qui sont vulnérables. C'est toujours un sujet particulier (individuel ou collectif) de droit qui peut souffrir de domination et qui peut, à l'inverse, réaliser sa liberté. Voir notre dernière partie. Voir aussi Pettit, "The Normative Analysis of the State», p. 7-10.

25. Pour ce qui est d'un «individualisme méthodologique» - qui prétend qu'il n'existe pas réellement d'entités sociales ou encore que ces entités sont réductibles à des unités non sociales - la compréhension républicaine des entités sociales (comme la nation) définit ces concepts comme des "propriétés sociales" présupposées dans toute existence communautaire. Cette position reste neutre quant au niveau du déterminisme impliqué dans la constitution individuelle de l'identité par exemple. Elle n'implique que la reconnaissance de l'existence de ces propriétés collectives sans se prononcer sur son impact psychologique réel sur les individus. (Pettit Ibid., p. 5-7)

26. Seymour, "Qui a peur des droits collectifs ?", p. 44. Voir aussi Anaya, Indigenous People in International Law, pp. 77-78.

27. Voir Pettit, Republicanism, p. 96-97.

28. Voir Tully, "The Struggles of Indigenous Peoples for and of Freedom», p. 56.

29. Cette position est tenue encore aujourd'hui par de nombreux philosophes libéraux au Canada, comme par exemple Daniel Weinstock. (Pour une critique sévère de cette position individualiste, voir l'article de Charles Blattberg, "L'hébreu en Israël : des leçons pour le français au Québec?", Argument, vol. 5, no 1, p.3-14.) L'exemple du Canada est pourtant, à ce sujet, probant : il a les moyens et la volonté d'une intervention arbitraire. Les pouvoirs de dépenser du fédéral, les pouvoirs excessifs du parti majoritaire à la Chambre des communes ainsi que son contrôle juridique par nomination, sont quelques-uns des aspects de ce régime qui posent d'importants problèmes dans une perspective républicaine. Par exemple, la Cour suprême du Canada s'est à plusieurs reprises attaquée aux droits linguistiques québécois. II est difficile, dans ce contexte, de comprendre la logique de la position de certains libéraux!

30. La complicité fréquente des tribunaux et des gouvernants est bien connue. Voilà pourquoi il est important que plusieurs instances du pouvoir soient en mesure d'intervenir lorsque la loi ne suffit pas. Au Canada, le Sénat n'a pas ce pouvoir et il est nommé par l'exécutif... La centralisation du pouvoir, le peu de place laissée aux citoyens dans les prises de décision et l'allure de propagande que prennent parfois les actions gouvernementales, sont totalement anti républicaines. (Voir à ce sujet la critique de Marc Chevrier : "De la monarchie en Amérique", L'Action nationale, vol. LXXXVIII, 5, mai, 1998, p. 90-129.) 
31. Ce que Philip Pettit appelle une "Electoral-cum-contestatory conception of Democracys, donc une démocratie qui ne s'appuie pas seulement sur l'élection de représentants politiques, mais aussi sur la contestation préférablement institutionnalisée des citoyens : une prise en charge par ces derniers d'une part de la responsabilité du respect des droits et des libertés («Minority Claims under Two Conceptions of Democracy», p. 204-215).

32. On peut penser aux législations coloniales ou à d'autres formes d'indirect rule. Des problèmes d'abus de pouvoir ou de non-respect des droits reconnus aux groupes surviennent souvent lorsque le pouvoir central a un intérêt différent de l'intérêt des membres de ses minorités. Les Canadiens-français, puis les Québécois, ont vécu historiquement cette situation à plusieurs reprises avec les gouvernements britannique et fédéral canadien.

33. Voilà une constante de la tradition républicaine : "Classical republicans theorists stressed that the constraint that laws impose on an individual's choices is not a restriction of liberty but an essential element of political liberty itself. They also believed that restrictions imposed by the law on actions of rulers as well as of ordinary citizens are the only valid shield against coercion on the part of any person or persons." Voir M. Viroli, Republicanism, Hill \& Wang, 2002, p. 9.

34. C'est là une différence fondamentale : "What the neo-roman writers repudiate avant la lettre is the key assumption of classical liberalism to the effect that force or the coercive threat of it constitute the only forms of constraint that interfere with individual liberty. The neo-roman writers insist, by contrast, that to live in a condition of dependence is in itself a source and a form of constraint. (...) This is why they insist (...) that to live in such a condition is to suffer a diminution not merely of security for your liberty but of liberty itself." (Skinner, Liberty Before Liberalism, p. 84.)

35. Voir Seymour, "Quelques aspects politiques de l'anti-individualisme», p. 64-65.

36. Stéphane Paquin, La revanche des petites nations, p. 198.

37. Philip Pettit, Republicanism, p. 52-55.

38. Les exemples de Pettit : force physique, avantages techniques et financiers, autorité politique, connections sociales, hiérarchie communautaire, information, positions idéologiques, légitimations culturelles, etc. Ibid., p. 57-59.

39. Ibid., p. 60.

40. Voir Rousseau, Des lois, in CEuvres complètes, Gallimard, 1964, vol. 3, p. 492 . Voir aussi Viroli, Republicanism, p.12.

41. Voilà aussi pourquoi Pettit affirme qu'il faille évaluer les institutions dans une perspective conséquentialiste : dans l'optique d'une maximisation de l'étendue et de l'intensité de la non-domination (Ibid., p. 97-109). II ne s'agit plus seulement alors de respecter des principes généraux, mais de s'attacher à la promotion d'un mode de vie libre et égalitaire.

42. Ibid., p. 50.

43. Ibid., p. 67-68.

44. II est à noter que tous les principes mis de l'avant par Pettit sont aussi applicables au niveau collectif. Pour cette précision, voir Republicanism, Ibid., Appendix, chap II, p.78-79. Voir aussi Skinner, Liberty Before Liberalism, p. 23-37.

45. Republicanism, p. 69. 
46. Ibid., p. 143-147. Pettit aborde directement la question des droits collectifs uniquement dans un article, où il reconnaît la légitimité de tels droits en renvoyant cependant au multiculturalisme de Kymlicka. II ajoute cependant que les droits des minorités seraient de facto mieux défendus dans une conception plus participative de la démocratie (et pas simplement électoraliste). II met de l'avant trois caractéristiques favorisant cet idéal : 1) l'imposition de restrictions formelles et informelles sur l'action gouvernementale; 2) certains aspects des décisions gouvernementales devraient au préalable être débattus publiquement et faire l'objet de consultations (surtout de la part de ceux qui seront les plus affectés par les propositions); 3) la possibilité de demander une révision juridique de la législation gouvernementale (par l'instauration de tels mécanismes accessibles aux citoyens). Selon Pettit, les libéraux conçoivent de telles mesures, déjà partiellement existantes, comme étant des limites (auto-)imposées à la démocratie ("majoritarienne»); or, dans la perspective républicaine, ces caractéristiques constituent en fait des aspects essentiels du régime démocratique lui-même. Voir : "Minority Claims under Two Conceptions of Democracy".

47. Republicanism, p.145.

48. bid. Voir aussi “Minority Claims...", p. 211-215.

49. Ibid., p.146.

50. Pour ce qui est de son importance démocratique : "lf we think that democracy requires a regime under which people have equal and full contestatory as well as electoral standing, then we should have no difficulty in seeing special minority claims as a natural part of the democratic package. We should have no hesitation about asserting that multicultural democracy is bound to make room for establishing such rights, whether at a minimal or at an intensive level." (Pettit, "Minority Claims...", p. 215)

51. Cette précision vise à contrer le problème de l'individualisme moral et politique.

52. Republicanism, p. 122-24. Voir aussi Viroli, Republicanism, p. 14.

53. Le Scottish National Party l'a compris depuis l'élection du gouvernement Thatcher au Royaume-Uni dans les années 70-80... II s'agit qu'un gouvernement soit opposé à l'autonomie politique d'une autre nation sur son territoire pour qu'une quantité de mesures soient soudainement votée, et que la centralisation de l'État reprenne (donc la radicalisation de la construction nationale). (Voir Paquin, La revanche des petites nations, p. 150-153) Le Québec a vécu la même situation avec les élections des gouvernements Trudeau et Chrétien (voir Kenneth McRoberts, Misconceiving Canada - The Struggle for National Unity, Oxford, 1997).

54. Republicanism, p. 124.

55. Pour le développement de l'auto-gouvernement au niveau de l'histoire des idées politiques, voir Vivienne Brown, "Self-Government: The Master Trope of Republican Liberty", The Monist, 84, 1, p. 60-76. 